\title{
Otimização Dinâmica Imuno-Inspirada para o Problema de Estimação de Direção de Chegada
}

\author{
Levy Boccato, Fabrício O. de França, Rafael Krummenauer, Romis Attux, Fernando Von Zuben e Amauri Lopes
}

\begin{abstract}
Resumo-O problema de estimação dos ângulos de chegada (DOA) de sinais incidindo em um arranjo de sensores, mediante o método de máxima verossimilhança, requer a otimização de uma função custo não-linear, não-quadrática, multimodal e variante com a relação sinal-ruído (SNR). Este trabalho apresenta os resultados obtidos por uma rede imunológica especializada em otimização multimodal e dinâmica, denominada dopt-aiNet. Simulações considerando cenários estáticos e dinâmicos foram realizadas. No primeiro caso, os resultados mostram que a doptaiNet consegue localizar com precisão o ótimo global, superando assim alguns métodos clássicos. No segundo caso, sua capacidade de rastrear o ótimo foi verificada e resultados promissores foram atingidos.
\end{abstract}

Palavras-Chave-Direção de chegada, estimação de máxima verossimilhança, otimização, sistemas imunológicos artificiais.

Abstract-The problem of estimating the direction of arrival (DOA) of signals impinging on an array of sensors, through the maximum likelihood method, requires the optimization of a cost function that is a non-linear, non-quadratic, multimodal and variant with the signal-noise ratio (SNR). This work presents the results obtained with an immune network specialized in multimodal and dynamic optimization, known as dopt-aiNet. Simulations were performed considering static and dynamic scenarios. In the former, the results obtained show that doptaiNet locates the global optimum with accuracy, overcoming some classical approaches. In the latter, its capability of tracking the optimum was assessed and promising results were achieved.

Keywords-Direction of arrival, maximum likelihood estimation, optimization, artificial immune systems.

\section{INTRODUÇÃO}

Os arranjos de sensores são estruturas comumente empregadas em uma série de aplicações, dentre as quais destacamse os sistemas de comunicações sem fio, sistemas de radar e sonar, além de sismologia [1]. A função dos sensores é coletar amostras da excitação que o ambiente exerce sobre o arranjo para que seja possível extrair informações espaçotemporais de sinais corrompidos por ruído. Uma informação de grande interesse é a localização da fonte emissora, a qual é determinada pelo ângulo de chegada ou DOA (Direction Of Arrival) [2].

De acordo com o método de estimação conhecido por máxima verossimilhança (em inglês, Maximum Likelihood (ML)),

Levy Boccato, Fabrício O. de França, Fernando Von Zuben e Romis Attux, Departamento de Engenharia de Computação e Automação Industrial, Faculdade de Engenharia Elétrica e de Computação, Universidade Estadual de Campinas, Campinas, Brasil, E-mails: lboccato@dca.fee.unicamp.br, olivetti@dca.fee.unicamp.br, vonzuben@dca.fee.unicamp.br, attux@dca.fee.unicamp.br. Rafael Krummenauer e Amauri Lopes, Departamento de Comunicações, Faculdade de Engenharia Elétrica e de Computação, Universidade Estadual de Campinas, Campinas, Brasil, E-mails:rkrummen@decom.fee.unicamp.br, amauri@decom.fee.unicamp.br. a estimativa do parâmetro é obtida a partir da maximização da função de verossimilhança, a qual, no caso do problema de DOA, possui um caráter não-linear, não-quadrático e multimodal, especialmente em condições críticas de relação sinalruído [2] [3]. Além disso, a superfície desta função varia consideravelmente à medida que a SNR é alterada, de maneira que a posição de seus pontos modais é modificada. Por conta destes fatos, somente uma abordagem de busca exaustiva é capaz de garantidamente localizar o ótimo global e, portanto, implementar o estimador ML com perfeição. Entretanto, por ser demasiadamente custosa, particularmente quando o número de sinais incidentes no arranjo aumenta, sua aplicação prática fica inviabilizada.

Alguns métodos foram propostos como alternativa à busca exaustiva [4] [5] [6]. Uma hipótese comum a todos eles é a ausência de variações no tempo dos cenários tratados, ou seja, durante sua execução, os parâmetros que descrevem o cenário permanecem inalterados. Contudo, mesmo com esta hipótese, estes algoritmos não conseguem atingir os resultados oferecidos por uma busca exaustiva.

Neste trabalho, propomos o uso de uma rede imunológica especializada em otimização multimodal e dinâmica, denominada dopt-aiNet [7], como alternativa aos métodos clássicos. Esta ferramenta possui a capacidade de preservar diversidade em sua população de soluções candidatas à medida que também realiza um refinamento local, o que a credencia como uma boa candidata para lidar com problemas de otimização dinâmica. De fato, a dopt-aiNet vem sendo empregada com notável sucesso em alguns problemas desta espécie, inclusive no contexto de telecomunicações [8].

O desempenho desta proposta será primeiramente analisado em termos de convergência global em um cenário estático consagrado na literatura DOA, no qual será possível confrontálo com os métodos clássicos. Posteriormente, variações dinâmicas em alguns parâmetros, como a SNR, serão introduzidas. Neste caso, avaliaremos a capacidade da dopt-aiNet rastrear o ótimo global e de se adaptar automaticamente às mudanças no ambiente.

Este trabalho está organizado da seguinte forma: Na Seção II, são definidos os modelos de sinal e ruído utilizados para descrever o problema de estimação DOA. Na Seção III, apresentamos o estimador ML para o problema de DOA, enquanto que na Seção IV destacamos os principais conceitos referentes à rede imunológica dopt-aiNet. Os resultados obtidos por este algoritmo considerando os cenários clássico e dinâmico se encontram na Seção V. Por fim, as principais conclusões e perspectivas futuras são apresentadas na Seção VI. 


\section{MODELO DE SINAL}

Considere $M$ ondas planas de banda estreita e mesma freqüência central incidindo em um arranjo linear uniforme de $N$ sensores. Os sensores estão igualmente espaçados com $d=\lambda / 2$, onde $\lambda$ é o comprimento de onda. As ondas incidem com ângulos $\phi=\left[\phi_{1} \ldots \phi_{M}\right]^{T}\left([\cdot]^{T}\right.$ denota o transposto), medidos em relação ao vetor normal ao eixo do arranjo. A Figura 1 exibe a geometria do arranjo e, em destaque, a $m$ ésima onda incidente.
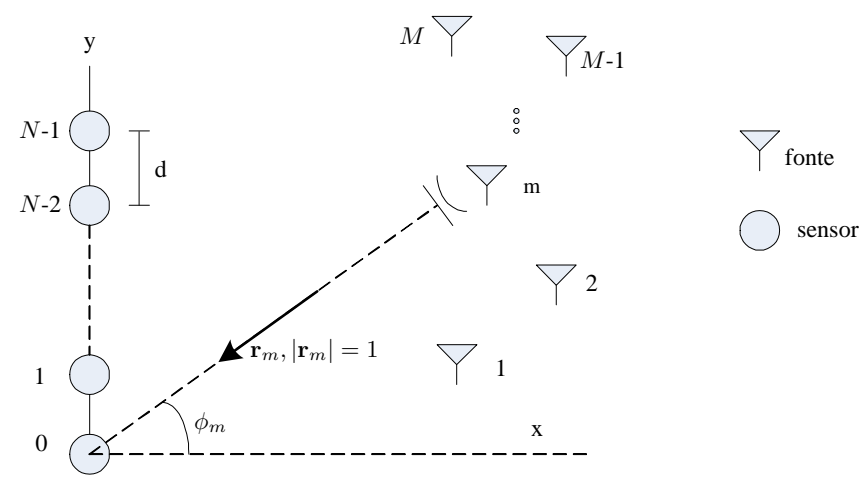

Fig. 1. Arranjo linear uniforme de $N$ sensores.

O problema de estimação DOA pode ser descrito pelo seguinte modelo matemático [4]:

$$
\mathbf{y}(k)=\mathbf{A x}(k)+\boldsymbol{\eta}(k), \quad k=1,2, \ldots, K
$$

onde $\mathbf{y}(k)=\mathbf{y}\left(t_{k}\right) \in \mathcal{C}^{N \times 1}$ representa o sinal recebido e amostrado no instante $t_{k}$ (snapshot), $\mathbf{x}(k) \in \mathcal{C}^{M \times 1}$ representa as amplitudes dos sinais recebidos, $\boldsymbol{\eta}(k) \in \mathcal{C}^{N \times 1}$ representa as amostras do ruído aditivo, $K$ é o número total de snapshots considerados, e $\mathbf{A}=\left[\mathbf{a}_{\mathbf{1}} \ldots \mathbf{a}_{\mathbf{M}}\right] \in \mathcal{C}^{N \times M}$, com $\mathbf{a}_{\mathbf{i}}=\left[1 e^{j \omega_{i}} \ldots e^{j(N-1) \omega_{i}}\right]^{T}$, é a matriz de resposta do arranjo, sendo que $\omega_{m}=\pi \sin \phi_{m}, m=1, \ldots, M$. O objetivo é estimar os ângulos de chegada $\phi_{m}$.

Admitimos que o número $M$ de fontes seja conhecido. Consideramos também que a matriz $\mathbf{A}$ possui posto cheio em colunas, implicando em $N \geq M$. O ruído $\boldsymbol{\eta}(k)$ é um processo aleatório circular complexo gaussiano estacionário de média zero, cuja matriz de covariância é expressa por $E\left\{\boldsymbol{\eta}(k) \boldsymbol{\eta}^{H}(i)\right\}=\sigma^{2} \mathbf{I} \delta_{k, i}$, onde $\delta_{k, i}$ é o operador delta de Kronecker, $E\{\cdot\}$ é o operador de média estatística ou esperança, $\{\cdot\}^{H}$ denota conjugado transposto de uma matriz ou vetor, e I denota a matriz identidade. O conjunto dos vetores de sinal $\mathbf{x}(k)$ corresponde a um processo aleatório circular complexo gaussiano estacionário de média zero, cuja matriz de covariância é dada por $E\left\{\mathbf{x}(k) \mathbf{x}^{H}(i)\right\}=\mathbf{C} \delta_{k, i}$. Os sinais são independentes do ruído.

É possível trabalhar com dois modelos para as fontes de sinal [9]: modelo condicional (CM), segundo o qual $\mathbf{y}(k) \backsim \mathcal{N}\left(\mathbf{A x}(k), \sigma^{2} \mathbf{I}\right)$, e o modelo incondicional (UM), no qual temos $\mathbf{y}(k) \backsim \mathcal{N}\left(\mathbf{0}, \mathbf{R}_{y}\right)$, onde

$$
\begin{aligned}
\mathbf{R}_{y} & =E\left\{\mathbf{y}_{k} \mathbf{y}_{k}^{H}\right\} \\
& =\mathbf{A C A}^{H}+\sigma^{2} \mathbf{I} .
\end{aligned}
$$

Para um número finito de snapshots, a matriz de covariância teórica $\mathbf{R}_{y}$ pode ser aproximada pela média amostral:

$$
\hat{\mathbf{R}}_{y}=\frac{1}{K} \sum_{k=1}^{K} \mathbf{y}_{k} \mathbf{y}_{k}^{H} .
$$

\section{Estimador de MÁXima VerossimilhançA}

O estimador ML é obtido por meio da maximização da função de verossimilhança [10]. Em [2] e [3], está demonstrado que, tendo em conta as hipóteses aqui adotadas, o estimador ML para o problema de DOA usando o modelo CM pode ser escrito como $^{1}$ :

$$
\hat{\boldsymbol{\theta}}_{M L}=\arg \min _{\boldsymbol{\theta}} \operatorname{Tr}\left\{\mathbf{P}_{\mathbf{A}}^{\perp} \hat{\mathbf{R}}_{y}\right\},
$$

onde $\operatorname{Tr}\{\cdot\}$ indica o traço de uma matriz, $\mathbf{P}_{\mathbf{A}}^{\perp}=\mathbf{I}-\mathbf{A A}^{\perp}$ é a matriz de projeção do subespaço de ruído e $\mathbf{A}^{\perp}=$ $\left(\mathbf{A}^{H} \mathbf{A}\right)^{-1} \mathbf{A}^{H}$ é a pseudo-inversa de $\mathbf{A}$.

A função custo

$$
J_{M L}(\boldsymbol{\theta})=\operatorname{Tr}\left\{\mathbf{P}_{\mathbf{A}}^{\perp} \hat{\mathbf{R}}_{y}\right\}
$$

é não-linear e não-quadrática em relação ao parâmetro $\theta$, e possui diversos mínimos locais. Com o propósito de ilustrarmos as características desafiadoras do problema de minimização da função custo $J_{M L}$, apresentamos na Figura 2 a superfície desta função considerando duas fontes de faixa estreita e de amplitudes unitárias incidindo no arranjo linear uniforme de 10 sensores com ângulos de chegada $\phi_{1}=10^{\circ}$ e $\phi_{2}=15^{\circ}$, para relações sinal-ruído de $0 \mathrm{~dB}$ e $-15 \mathrm{~dB}$. Nas duas situações, foram utilizados 100 snapshots. A matriz de covariância do sinal é dada por $\mathbf{C}=\mathbf{I}$, e a relação sinalruído, em $\mathrm{dB}$, é definida como $\mathrm{SNR}=10 \log 1 / \sigma^{2}$, onde $\sigma^{2}$ corresponde à variância do ruído.

A Figura 2 evidencia o impacto da relação sinal-ruído na superfície da função custo $J_{M L}$. Conforme reduzimos a SNR, o ponto de mínimo global se desloca, de modo que suas coordenadas não mais coincidem com os verdadeiros ângulos de chegada. Além disto, o caráter multimodal da função se torna cada vez mais pronunciado.

Estas duas evidências servem para ilustrar os desafios com os quais a rede dopt-aiNet terá de lidar. Como agravante, no caso do cenário dinâmico, poderão ocorrer mudanças na superfície da função custo, como aquelas apresentadas na Figura 2, enquanto a dopt-aiNet procura o ótimo global.

$\mathrm{Na}$ próxima seção, descrevemos os principais conceitos referentes à dopt-aiNet.

\section{UMA REDE IMUNOLÓGICA ARTIFICIAL PARA OTIMIZAÇÃO EM AMBIENTES DINÂMICOS}

A área de pesquisa caracterizada pelo estudo de sistemas imunológicos artificiais tem experimentado um crescente interesse nos últimos anos. Os sistemas imunológicos artificiais (em inglês, Artificial Immune Systems (AIS)) compõem um vasto repertório de sistemas adaptativos capazes de realizar

\footnotetext{
${ }^{1}$ Note que a variável $\boldsymbol{\theta}$ denota genericamente o parâmetro de direção de chegada, enquanto que $\phi$ representa os valores verdadeiros dos ângulos de chegada.
} 


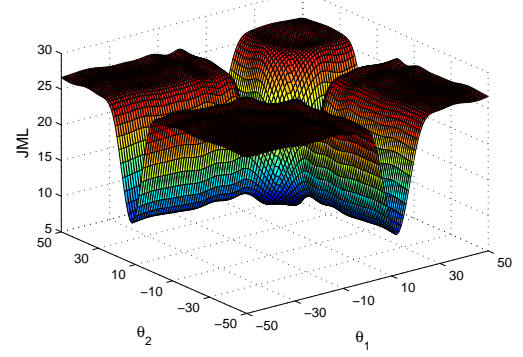

(a) $\mathrm{SNR}=0 \mathrm{~dB}$

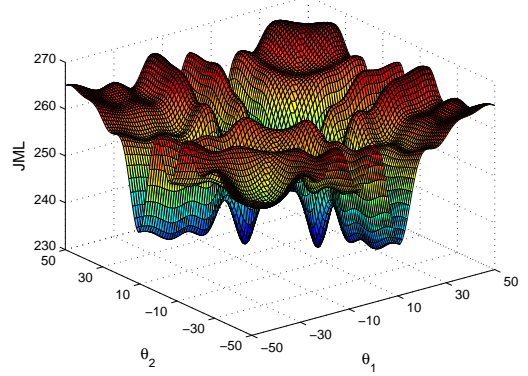

(b) $\mathrm{SNR}=-15 \mathrm{~dB}$

Fig. 2. Superfícies da função ML Condicional para $M=2, N=10$, 100 snapshots e relações sinal-ruído de $0 \mathrm{~dB}$ e $-15 \mathrm{~dB}$.

uma ampla gama de tarefas em algumas linhas de pesquisa, como reconhecimento de padrões, navegação autônoma e controle, análise de dados e otimização [11]. Apesar de sua diversidade, a maioria dos AIS compartilha algumas características interessantes:

- Controlam dinamicamente o número de indivíduos (células) na população.

- São inerentemente capazes de manter diversidade na população.

- Tendem a preservar soluções localmente ótimas quando as encontram.

Estas habilidades tornam os AIS excelentes candidatos para lidar com problemas de otimização, e, de fato, alguns algoritmos imuno-inspirados já foram desenvolvidos para este propósito [12]. Em [13], uma rede imunológica, denominada opt-aiNet, foi desenvolvida para lidar com problemas de otimização em contextos multimodais. Tal rede emprega os princípios de seleção clonal [14] e maturação de afinidade [15] em conjunto com a teoria da rede imunológica [16] de modo a estabelecer um elegante compromisso entre exploração e explotação do espaço de busca.

Em [7], a opt-aiNet foi aprimorada e estendida para lidar com ambientes dinâmicos. O algoritmo modificado, denominado dopt-aiNet (artificial immune network for dynamic optimization), está esquematizado no Quadro 1.

Em primeiro lugar, o algoritmo gera aleatoriamente uma população inicial de células (isto é, uma matriz com $N_{i}$ vetores solução (células) de dimensão $M$, onde $M$ é o número de ângulos de chegada). Em seguida, o algoritmo entra no laço principal, onde cada célula é avaliada e gera $N_{c}$ clones, os quais são cópias exatas da célula original. Para cada clone,

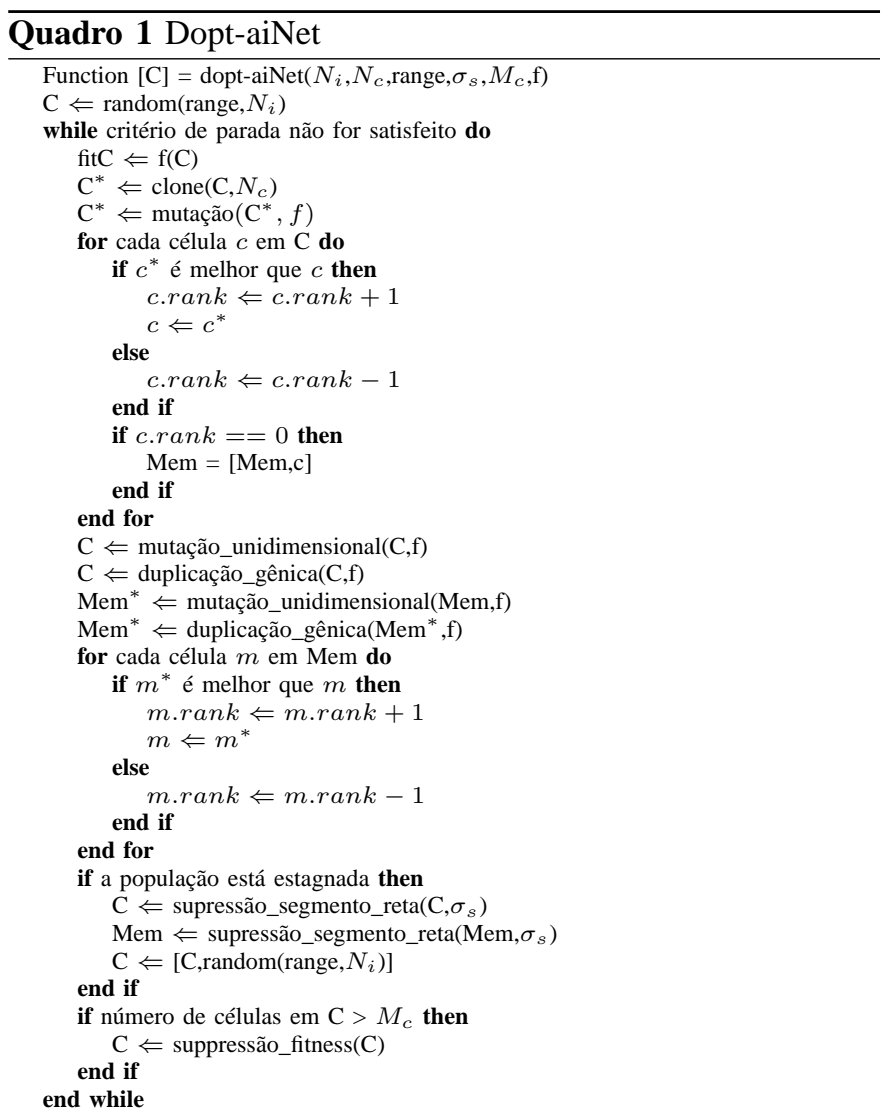

uma mutação é realizada por meio da definição de um vetor real G gerado aleatoriamente por uma distribuição gaussiana, de média zero e desvio padrão unitário, e que representa a direção do movimento de cada clone. Adicionalmente, o tamanho do passo a ser dado na direção definida por $\mathbf{G}$ é determinado com o auxílio de um algoritmo de busca unidimensional denominado seção áurea, o qual otimiza o passo $\alpha$ na direção $\mathbf{G}$ (ou seja, $f(x+\alpha \mathbf{G})$ ). Deste modo, o clone mutado é obtido por

$$
\mathbf{c}^{*}=\mathbf{c}+\alpha \mathbf{G} \text {. }
$$

Com a finalidade de reduzir o custo computacional, cada célula recebe um valor denominado rank, o qual é decrementado toda vez que um operador de mutação não é capaz de melhorar o valor custo associado à célula original. Por outro lado, quando a mutação gera um clone superior à célula original, o rank é incrementado. Caso este valor chegue a zero, a célula é transferida para uma população de memória, a qual é tratada somente com dois operadores especiais de mutação que serão descritos a seguir. Quando nenhuma melhoria é possível de ser obtida para uma determinada célula, esta permanecerá inalterada durante as iterações restantes.

Após este passo, as células são modificadas através de dois novos operadores de mutação propostos em [7]: a mutação unidimensional e a duplicação gênica. A mutação unidimensional trata uma direção por vez, de modo a realizar uma busca mais fina na vizinhança da célula. As direções são definidas por uma matriz identidade e pelos vetores unitários $+\mathbf{1}$ (vetor com todos os elementos iguais a 1) e -1. O processo de duplicação gênica 
substitui o valor de um certo elemento da célula pelo valor de outro elemento escolhido aleatoriamente caso esta ação traga melhorias no valor do fitness. Quando este processo termina, esta nova célula é introduzida na população.

Caso a população apresente estagnação, a similaridade entre as células é calculada e, quando duas células são muito similares entre si, aquela com pior fitness é suprimida. Após a supressão, novas células são geradas aleatoriamente e introduzidas na população.

O algoritmo denominado Supressão por Distância de Segmento de Reta procura estimar a posição relativa de cada par de células por meio da comparação entre os valores da função em pontos intermediários e os valores produzidos por um segmento de reta conectando as células sob análise. Para quaisquer dois pontos definidos pela célula e seu valor de fitness, por exemplo, $P 1=(c 1, f(c 1))$ e $P 2=(c 2, f(c 2))$, o ponto médio é obtido e avaliado. Se a distância entre o terceiro ponto $P 3=((c 1+c 2) / 2, f(c 1+c 2 / 2))$ e o segmento de reta que liga os pontos $P 1$ e $P 2$ for menor ou igual a um limiar de supressão $\sigma_{s}$, então a célula com pior fitness é eliminada. A principal vantagem deste método de supressão em relação àquele utilizado pela opt-aiNet [13] é que a escolha de um valor para o limiar de supressão é bem mais tratável. Considerando testes em diversas funções, os autores em [7] sugerem o valor $\sigma_{s}=0,5$.

Finalmente, se o número de células existentes na população ultrapassar um limite pré-estabelecido $\left(M_{c}\right)$, os piores indivíduos são eliminados. Isto tende a reduzir o custo computacional do método sem comprometer seu desempenho.

\section{Resultados e AnÁlise}

Nesta seção, apresentamos os principais resultados obtidos pela rede dopt-aiNet no problema de estimação DOA. Primeiramente, será considerado o cenário estático descrito na Seção III, o qual é empregado como uma referência de teste para métodos de estimação DOA e está presente em diversas publicações da área, como em [4], [5], [6]. O objetivo é verificar a qualidade da estimação realizada pela dopt-aiNet para então compará-la com os algoritmos MODE e MODEX, além da busca exaustiva, a qual estabelece a referência de desempenho para métodos baseados no critério ML.

A qualidade da estimação dos ângulos de chegada será avaliada por meio da raiz quadrada do erro quadrático médio (RMSE), expressa por

$$
R M S E=\sqrt{\sum_{i=1}^{M} \sum_{j=1}^{P} \frac{\left(\phi_{i}-\theta_{i j}\right)^{2}}{M P}},
$$

onde $\phi_{i}$ é o ângulo de chegada do $i$-ésimo sinal incidente, $\theta_{i j}$ é a estimativa do $i$-ésimo ângulo de chegada no $j$-ésimo experimento, $M$ é o número de fontes e $P$ representa o número de experimentos, isto é, o número de estimativas dos ângulos de chegada que consideramos para uma dada relação sinalruído, mantendo o sinal fixo e variando a realização de ruído. Em todas as simulações, utilizaremos $P=1000$.

Posteriormente, analisaremos o desempenho da dopt-aiNet em um cenário dinâmico, no qual variações em alguns parâmetros serão efetuadas durante sua execução.

\section{A. Cenário Estático}

Os valores atribuídos aos principais parâmetros da rede dopt-aiNet considerando o cenário descrito na Seção III são: $N_{i}=20, N_{c}=5, M_{c}=60$ e $\sigma_{s}=0,5$. O critério de parada adotado foi um número máximo de iterações igual a 250. Além disto, uma vez que todos os ângulos de chegada são distintos por hipótese, preferimos não empregar o operador de duplicação gênica, o qual dificilmente traria progressos significativos à população de células.

Empregando o conjunto de valores explicitado anteriormente, foi possível obter a curva de RMSE em função da relação sinal-ruído para a dopt-aiNet, para os métodos MODE, MODEX e busca exaustiva, além do limite de Cramer-Rao. A Figura 3 apresenta os resultados.

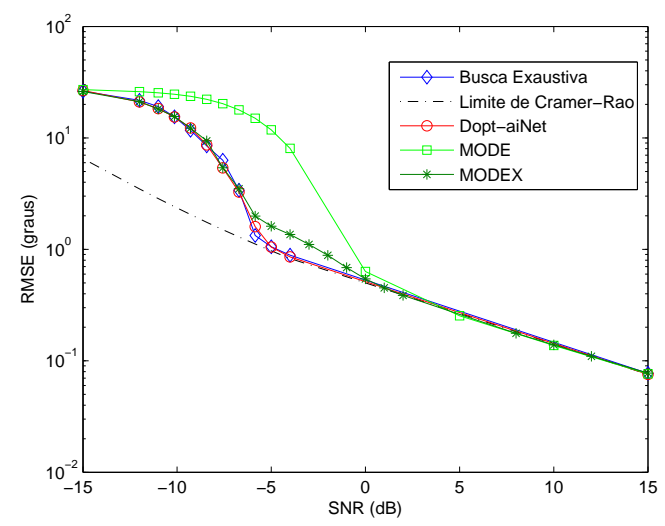

Fig. 3. Curva de RMSE em função da SNR.

Observando a Figura 3, é possível perceber que, ao longo de toda a faixa de valores de SNR, a rede dopt-aiNet foi capaz de acompanhar o desempenho da busca exaustiva. As pequenas discrepâncias existentes decorrem, fundamentalmente, do número de simulações utilizado e também do conjunto de valores definido para os parâmetros da dopt-aiNet. Isto significa que esta ferramenta conseguiu implementar o método de estimação ML para o problema de estimação DOA. Observe ainda que esta ferramenta superou os desempenhos obtidos pelos algoritmos MODE e MODEX, especialmente na região de limiar, quando estes se afastam do limite de Cramer-Rao.

\section{B. Cenário Dinâmico}

Neste cenário, dois fatores relevantes são aleatoriamente alterados, segundo uma distribuição uniforme, enquanto a dopt-aiNet realiza a otimização da função custo $J_{M L}$ : a relação sinal-ruído, que assume valores entre -15 e $15 \mathrm{~dB}$, e os ângulos de chegada, os quais admitem valores no intervalo $\left(-90^{\circ}, 90^{\circ}\right)$. Optamos por introduzir variações em degrau nestes parâmetros pelo fato de representarem os perfis mais desafiadores de variação, sendo mais abruptos, por exemplo, que uma variação em rampa.

Além disto, estas modificações serão realizadas a cada 25 iterações. Este valor foi assim definido uma vez que se mostrou adequado a ponto de permitir a análise da velocidade de reação do algoritmo às mudanças no ambiente ao mesmo tempo que 
concede à dopt-aiNet uma chance de se adaptar à nova situação e de refinar a população.

Consideramos novamente apenas 2 fontes emissoras. Isto é intessante, pois possibilita a execução de uma rotina de busca exaustiva para localizar o ótimo global após cada modificação no cenário, o que por sua vez facilita a avaliação do desempenho da dopt-aiNet, dado que podemos comparar a evolução da estimativa DOA fornecida pela dopt-aiNet com aquela determinada pelo ótimo global.

Os seguintes valores foram atribuídos aos parâmetros da dopt-aiNet: $N_{i}=20, N_{c}=10, M_{c}=80$ e $\sigma_{s}=0,5$. Após 500 iterações, o algoritmo é encerrado. A Figura 4(a) apresenta a curva com a evolução da distância do melhor indivíduo em relação à posição do ótimo global.

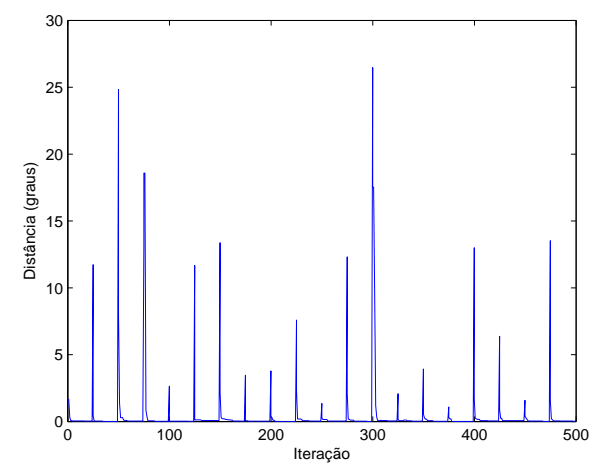

(a) distância em relação ao ótimo

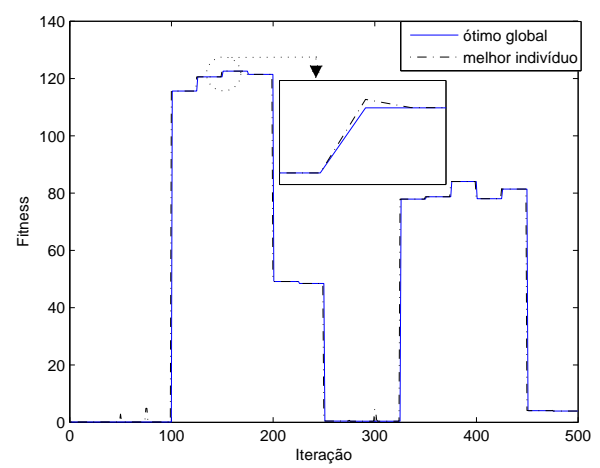

(b) Evolução do fitness do melhor indivíduo

Fig. 4. Distância em relação ao ótimo e fitness do melhor indivíduo.

Dois fatos interessantes podem ser observados na Figura 4(a): 1) imediatamente após uma alteração em algum dos parâmetros do cenário, a distância do melhor indivíduo em relação ao ótimo pode aumentar significativamente, o que indica que a superfície da função custo foi bastante alterada. 2) mesmo nos casos em que as mudanças foram mais pronunciadas, a dopt-aiNet foi capaz de rapidamente localizar a nova posição do ótimo global.

Estes dois fatos também podem ser verificados na Figura 4(b), a qual apresenta a evolução do fitness do melhor indivíduo e do ótimo global ao longo das iterações. O quadro em destaque na Figura 4(b) amplia as curvas de fitness para melhor visualização de seus comportamentos. Observe que imediatamente após uma alteração no cenário, o fitness do melhor indivíduo pode ficar um pouco mais distante do valor de fitness do ótimo global. Entretanto, rapidamente converge para este último, o que ressalta a capacidade que a dopt-aiNet possui de rastrear o ótimo global.

As Figuras 5 e 6 mostram, respectivamente, a evolução da estimativa de cada ângulo referente ao melhor indivíduo e ao indivíduo mais próximo ao ótimo global. Elas são motivadas pelo fato de, após o cenário ser alterado, o indivíduo mais próximo ao ótimo nem sempre ser aquele de melhor fitness, especialmente quando consideramos as SNRs baixas.

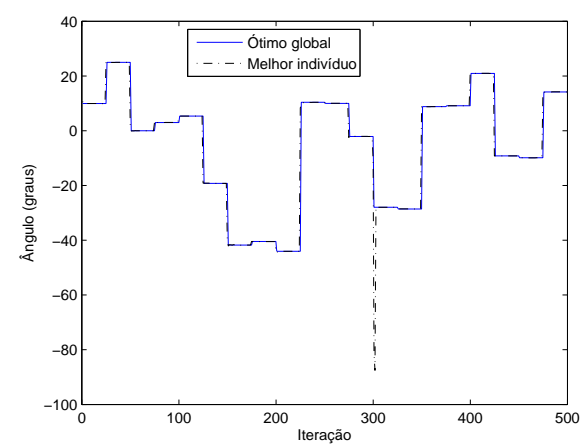

(a) Primeiro ângulo

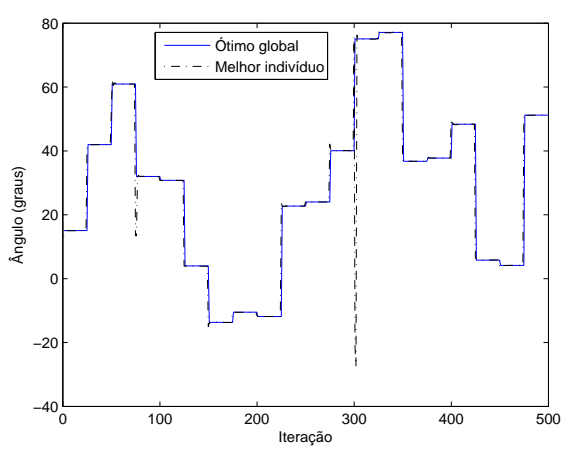

(b) Segundo ângulo

Fig. 5. Estimativas associadas ao melhor indivíduo.

Observando a Figura 5, é possível notar que, após algumas variações no cenário, o melhor indivíduo da população pode estar bem distante do ótimo global. Contudo, após poucas iterações, já está situado bem próximo ao ótimo global, o que indica que a dopt-aiNet conseguiu se adaptar ao novo ambiente de otimização e identificou a região onde o ótimo está situado.

A Figura 6 corrobora as observações citadas anteriormente e também destaca outro aspecto importante: mesmo após as mudanças mais abruptas em algum dos parâmetros que descrevem o cenário, existe um indivíduo razoavelmente próximo à nova posição do ótimo. Isto se deve à capacidade que a dopt-aiNet possui de manter diversidade na população, o que, conforme já enfatizado, é extremamente interessante em contextos de otimização dinâmica.

Em suma, os resultados apresentados nesta seção mostram o grande potencial que a dopt-aiNet possui de se adaptar às modificações que a função custo sofre quando um dos parâmetros que descrevem o cenário DOA é alterado. Além disto, foi verificado que em poucas iterações, a dopt-aiNet 


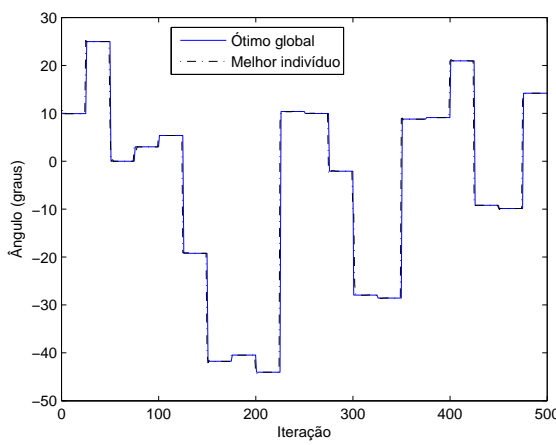

(a) Primeiro ângulo

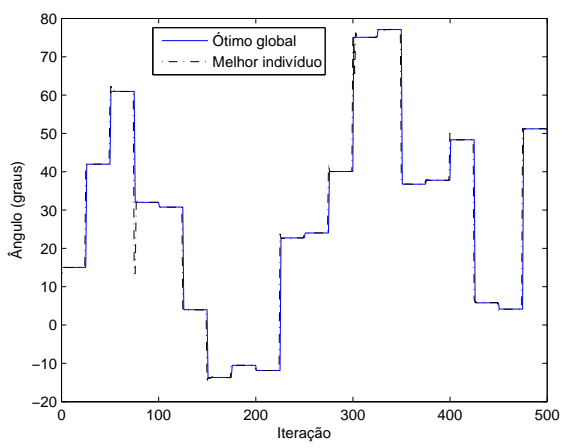

(b) Segundo ângulo

Fig. 6. Evolução das estimativas - indivíduo mais próximo ao ótimo.

localiza a nova posição do ótimo global, ao mesmo tempo que posiciona outros indivíduos em regiões localmente ótimas do espaço de busca, o que aumenta suas chances de responder rapidamente a outras variações no cenário.

\section{CONCLUSÕES}

O problema de estimação de direção de chegada conduz, por meio do critério ML, a um problema de minimização de uma função custo $J_{M L}$ de caráter não-linear, não-quadrático, multimodal e variante com a relação sinal-ruído. Neste trabalho, aplicamos uma rede imunológica adaptada para ambientes multimodais e dinâmicos, conhecida como dopt-aiNet, para realizar a estimação DOA.

Simulações no contexto de um cenário estático clássico na literatura mostraram que a dopt-aiNet determina com precisão o mínimo global da função custo $J_{M L}$ para uma ampla faixa de valores de relação sinal-ruído. Neste sentido, o desempenho deste algoritmo praticamente se iguala àquele oferecido pelo método de busca exaustiva e supera alguns métodos tradicionais, como MODE e MODEX, pois estes últimos falham em determinar o mínimo global para um conjunto de valores de SNR, especialmente abaixo da região de limiar, conforme já evidenciado na literatura.

Além disto, foi possível verificar o desempenho da doptaiNet em um cenário dinâmico, no qual os ângulos de chegada e a relação sinal-ruído variaram. Os resultados evidenciaram a grande capacidade de rastreamento do ótimo global e de adaptação a um novo ambiente que a dopt-aiNet possui. Por conta disto, boas perspectivas se abrem a partir deste estudo, entre as quais destacamos: 1) análise de desempenho em cenários com mais fontes; 2) avaliação mais detalhada do custo computacional da proposta; 3) possibilidade de incorporar conhecimento específico do problema DOA à própria doptaiNet ou a algum operador; 4) possibilidade de considerar entrada e saída de fontes, além de modificações no cenário de snapshot em snapshot, isto é, à medida que as amostras são coletadas. Por fim, também parece ser uma perspectiva interessante estender a aplicação da dopt-aiNet a outros contextos dinâmicos em telecomunicações, como os que ocorrem quando se lida com rádio cognitivo [17].

\section{AGRADECIMENTOS}

Este trabalho contou com o apoio da FAPESP, processo $n^{\circ}$ 08/56937-2, e também do CNPq e CAPES.

\section{REFERÊNCIAS}

[1] S. Haykin, Array Signal Processing. Englewood Cliffs, NJ: Prentice Hall, 1985.

[2] H. L. Van Trees, Optimum Array Processing. Part IV of Detection, Estimation and Modulation Theory. New York, USA: John Wiley and Sons, 2001.

[3] R. Krummenauer, "Filtragem ótima na estimação de direção de chegada de ondas planas usando arranjo de sensores," Dissertação de Mestrado, Faculdade de Engenharia Elétrica e Computação - UNICAMP, Campinas, SP, Jul. 2007.

[4] P. Stoica e K. C. Sharman, "Novel eigenanalysis method for direction estimation," IEE Proceedings part F (Radar and Signal Processing), vol. 137, $\mathrm{n}^{\circ}$. 1, pags. 19-26, fev 1990.

[5] A. Gershman e P. Stoica, "MODE with extra-roots (MODEX): a new DOA estimation algorithm with an improved threshold performance," em IEEE International Conference on Acoustics, Speech, and Signal Processing, vol. 5, Phoenix, AZ, Mar. 1999, pags. 2833-2836.

[6] A. Lopes, I. S. Bonatti, P. L. D. Peres, e C. A. Alves, "Improving the MODEX algorithm for direction estimation," Signal Processing, vol. 83, $\mathrm{n}^{\circ}$. 9, pags. 2047-2051, set 2003.

[7] F. O. de França, F. J. Von Zuben, e L. N. de Castro, "An artificial immune network for multimodal function optimization on dynamic environments," em Proceedings of the Genetic and Evolutionary Computation Conference (GECCO), vol. 1, Washington D.C., 2005, pags. 289-296.

[8] C. Junqueira, F. O. de França, R. R. F. Attux, C. M. Panazio, L. N. de Castro, F. J. Von Zuben, e J. M. T. Romano, "Immune-inspired dynamic optimization for blind spatial equalization in undermodeled channels," em Proceedings of the IEEE Congress on Evolutionary Computation, vol. 1, 2006, pags. 9801-9808.

[9] P. Stoica e A. Nehorai, "Performance study of conditional and unconditional direction-of-arrival estimation," IEEE Transactions on Acoustics, Speech, and Signal Processing, vol. 38, n ${ }^{\circ}$ 10, pags. 1783-1795, 1990.

[10] S. M. Kay, Fundamentals of Statistical Signal Processing, Volume I: Estimation Theory. Englewood Cliffs, NJ: Prentice Hall Signal Processing Series, 1993.

[11] L. N. de Castro e J. Timmis, Artificial Immune Systems: A New Computational Intelligence Approach. London, Great Britain: Springer, 2002.

[12] L. N. de Castro e F. J. Von Zuben, "Learning and optimization using the clonal selection principle," IEEE Transactions on Evolutionary Computation, vol. 6, $\mathrm{n}^{\circ}$. 3, pags. 239-251, 2002.

[13] L. N. de Castro e J. Timmis, "An artificial immune network for multimodal function optimization," em Proceedings of IEEE Conference on Evolutionary Computation, vol. 1, 2002, pags. 699-704.

[14] F. M. Burnet, Clonal Selection Theory of Acquired Immunity. Cambridge Univ. Press, 1959.

[15] G. L. Ada e G. J. V. Nossal, "The clonal selection theory," Scientific American, pags. 50-57, 1987.

[16] N. K. Jerne, "Towards a network theory of the immune system," Ann. Immunol. (Inst. Pasteur), pags. 373-389, 1974.

[17] J. I. Mitola, "Cognitive radio for flexible mobile multimedia communications," em IEEE International Workshop on Mobile Multimedia Communications, San Diego, CA, nov 1999, pags. 3-10. 